\title{
Towards a Comprehensive Model for E-Government Adoption and Utilisation Analysis: The Case of Saudi Arabia
}

\author{
Saleh Alghamdi \\ University of Sussex, \\ Informatics Department \\ Brighton, UK \\ Email: sa434@sussex.ac.uk
}

\author{
Natalia Beloff \\ University of Sussex, \\ Informatics Department, \\ Brighton, UK \\ Email: N.Beloff@sussex.ac.uk
}

\begin{abstract}
E-Government increases transparency and improves communication between the government and the users. However, users' adoption and usage is less than satisfactory in many countries, particularly in developing countries. This is a significant factor that can lead to e-Government failure and, therefore, to the waste of budget and effort. Unlike much research in the literature that has utilised common technology acceptance models and theories to analyse the adoption of e-Government, which may not be applicable for e-Government acceptance analysis, this study proposes a more comprehensive and appropriate framework for analysing the significant factors that could influence the adoption and utilisation of e-Government in Saudi Arabia, as this is becoming a necessity.
\end{abstract}

\section{INTRODUCTION}

$\mathbf{I}$ NFORMATION and Communication Technologies (ICTs) are considered to be the backbone of many activities used nowadays. They have tremendous potential to provide solutions and to solve problems in different aspects, which then leads to enhanced quality of life. Given the fact that the accelerated development of Information Technologies (IT) has led to a rapid increase in the number of websites and services provided by governments, nearly all governments of countries around the world have at least a web presence, or so-called e-Government [1]. Currently, the role of ICTs is crucial in governance processes, where they can help to create a structured network for service delivery [2], effectiveness and efficiency [3], interactivity, accountability and transparency [4].

The term e-Government has various definitions in the literature [5] [6], most of which refer only to providing online services (E-Services) to citizens, whereas the definition of e-Government is, in fact, broader and more comprehensive. Although defining e-Government from a single perspective is easy, defining it from general view is relatively difficult [7]. However, we can provide a more comprehensive definition, namely the utilisation of various Information and Communication Technologies (ICTs) for facilitating communication between the government and the stakeholders (citizens, businesses and governmental agencies), providing effective, efficient and integrated e-Services that enhance the relationship between the government and the stakeholders through multiple and flexible channels, leading to a more democratic system and increased engagement.

The field of Electronic Government is growing considerably; therefore, many research areas need to be studied in order to provide scientific insights [8]. One of the most important elements of implementing E-Government systems is the interaction between users and E-Government systems, specifically the adoption and utilisation by users, who are the main target when implementing such systems. This interaction element is considered the main method for measuring the utilisation and success of E-Government systems. If there is no interaction between users and E-Government systems, this means that there is no benefit of implementing such systems. Therefore, this research will investigate and analyse factors that could influence the adoption and utilisation of e-Government in Saudi Arabia from different perspectives, which will then lead to better understanding, ensuring that e-Government has a high level of success.

This paper is divided into five sections. The first introduced the proposed definition of e-Governance. The second section provides a literature review and background information regarding e-Government in Saudi Arabia. The third section explains the research model. The fourth section provides a brief discussion of the research model and the development thereof. The final section provides a conclusion and details our planned future work to utilise, test and validate the proposed model.

\section{BACKGROUND AND CONTEXT}

\section{A. Literature Review}

E-Government initiatives are still in the early stages in most developing countries, and face many issues related to adoption, implementation and utilisation. Users' adoption of e-Government systems is less than satisfactory in many countries, particularly in developing countries. Although large amounts of money have been invested in e-Government initiatives in some of these countries, such as the Arab countries, they still have many challenges and shortcomings that slow the adoption and minimise the utilisation of e-Government 
systems, which influence the success of such systems [9]. Most of the studies in the literature have focused on implementing e-Government from technical and structural perspectives [10]. Several studies also focused mainly on analysing barriers to and challenges of implementing e-Government [11]. However, few recent studies have been conducted to discover and analyse factors that can affect the adoption and utilisation of eGovernment from the users' perspective [12] [2] [13].

Several models and theories in the literature have been developed to study the acceptance and the diffusion of technologies, including the Theory of Reasoned Action (TRA), the Technology Acceptance Model (TAM), the Diffusion of Innovation Theory (DOI), Perceived Characteristics Innovation (PCI) and the Unified Theory of Acceptance and Use of Technology (UTAUT) [14][15][16][17][18]. Most of the research in e-Government literature has utilised some of these common models and theories to analyse the adoption of eGovernment, either by using their original forms, by adding certain constructs to them or by combining them. However, some of the models that have been used for analysing the adoption of e-Government in the literature were critically analysed in this research in order to evaluate their applicability for studying levels of e-Government's adoption and utilisation. This will help us to fill the gaps and to overcome shortcomings, which exist in the conducted studies, while developing this research model.

Some significant constructs involved in the analysed models are important and are supported in the literature; thus, they will be integrated into the current model. However, certain other constructs are not supported in the literature, which means they are not significant and will therefore not be used for analysing the adoption and utilisation of e-Government. Furthermore, a number of significant factors that are likely to have influence on the adoption and the usage of e-Government were not addressed in some of the analysed models. Table (I) shows some of the reasons that certain commonly used models are not applicable to the analysis of the adoption and usage of e-Government.

As stated previously, some recent studies in the literature have analysed e-Government adoption, and most have utilised the common models that are given in Table (1) [2] [22]. Therefore, the outcomes were limited due to the limitations that exist in the frameworks utilised. Several studies have amended the original forms by adding extra constructs, such as risk and trust [23] [12] [24], or by combining some common models [19].

\section{B. E-Government in Saudi Arabia}

The Saudi e-Government programme 'Yesser' was officially launched in 2005 [25]. Yesser is an Arabic word that means 'make it easy'. Consequently, the Yesser programme aims to provide services and information to citizens easily [26]. It serves as an enabler and facilitator for transforming the public sector to the information society, whereas each governmental agency is responsible for the actual execution of its own website and is responsible for effective coordination
TABLE I: Inapplicability of common models for the eGovernment context

\begin{tabular}{ll}
\hline $\begin{array}{l}\text { The } \\
\text { Model }\end{array}$ & Inapplicability for the e-Government context \\
\hline & - The Subjective Norm construct is one of least understood \\
aspects of TRA [15]. The Subjective norm $(S N)$ construct is \\
likely to have an indirect impact on Behavioural Intention \\
$(B I)$ via the Attitude towards a behaviour $(A)$ construct. This \\
will make the differentiation between the direct effect of $S N$ \\
TRA & on BI and the indirect effect of $S N$ on $B I$ via $A$ more difficult. \\
& There is a lack of significant constructs that can analyse the \\
adoption and utilisation of large and complex systems such & as e-Government.
\end{tabular}

- External Variables that have been proposed as an influential factor that affect the Perceived Ease of Use (PEU) and Perceived Usefulness $(P U)$ are not fully explored in TAM [19].

- Attitude towards using does not fully mediate Perceived Ease of Use (PEU) and Perceived Usefulness (PU) [15].

- TAM cannot capture and specify the complete essence of eGovernment usage behaviour [20], due to the lack of many important factors and constructs that have direct impact on behaviour in term of intention to use, as well as on the actual usage of technologies, particularly e-Government systems.

- TAM excluded some important resources of variance, and did not consider other important factors that could prevent users from using information systems, such as time and money constraints [2].

- The Trialability construct, which refers to how easily a new technology can be used experimentally, cannot be applied to certain technologies and systems, including e-Government, due to the nature of the data and the number of users; thus, such systems are not trialable. This does not, however, mean that testing and validating the system is not essential.

DOI - The Observability construct, which refers to the extent to which the innovation provides visible and tangible results, can be implicitly integrated into the Relative Advantage construct.

- DOI Model also lack certain factors and constructs that are crucial when analysing the diffusion and adoption of new technology, particularly e-Government.

- UTAUT did not address some very important constructs and factors, such as perceived awareness and quality of service,although these are highly likely to have a strong impact on behaviours and intentions to use and adopt technologies. It also did not address constructs related to reliability aspects such as security, privacy, trust and perceived regulations. Moreover, UTAUT did not address the influence of culture on the adoption and utilisation of technologies. Although UTAUT considered the influence

UTAUT of certain personal demographic factors, including age and gender, it did not address other important demographic factors, such as the user's location, the user's education level and the user's income, which are likely influence the utilisation and adoption level.

- UTAUT ignored the influence of some constructs on the others, although this is very important in such analyses.

- Grouping and labelling of items in UTAUT is problematic, since a variety of disparate items is combined to represent a single construct [21]. 
with the Yesser programme [27][28]. According to the eGovernment's first action plan document, the vision of the Saudi e-Government initiative is to provide better government services to users; thus, the vision statement can be summarised as [28]:

'By the end of 2010, everyone in the Kingdom will be able to enjoy from anywhere and at any time - world-class government services offered in a seamless, user-friendly and secure way by utilizing a variety of electronic means."

To achieve this vision, the Saudi e-Government aims to provide 150 top-priority services for citizens and residents by 2010 , and to make them available $24 / 7$ with a $75 \%$ usage level and an $80 \%$ user satisfaction rating [29]. Unfortunately, the Saudi e-Government's vision has not yet been completely realised, despite the fact that it is currently 2014 . An evaluation study was conducted by Al-Nuaim in 2011 to evaluate the Saudi ministries' websites, which were considered to be eGovernment service providers. This study indicated that nine Saudi ministries, which represent $41 \%$ of the evaluated ministries, did not implement a true e-Government website. Moreover, ten ministries, which represent $45.4 \%$ of the evaluated ministries, were completely or partially in the 'web presence' stage. Three ministries, which represent $13.6 \%$ of the evaluated ministries, were in the 'one-way interaction' stage [26]. Some improvements might have been achieved during the period between Al-Nuaim's study and the present, but we believe that this period is not sufficient to accomplish that which was expected by 2010, based on the vision statement.

The focus of Al-Nuaim's study was the evaluation of e-Government service providers' websites, which measure the achievement of the first important aspect of the vision of Saudi's e-Government, namely providing over 150 toppriority e-Services. However, the focus of our research work will be on the utilisation of e-Government from the user's perspective, which represents the second crucial part of the Saudi e-Government's vision (75\% usage level and $80 \%$ user satisfaction rating). This is a significant contribution of this research and is one that could lead to a better understanding of the factors that can influence the adoption and usage level of e-Government.

Unlike developed countries, research and studies that investigate and analyse the adoption and utilisation of e- Government in developing countries are limited. Few studies have been conducted on e-Government utilisation, e-Usage and eEngagement in any country, and this is particularly true of Arab countries, such as Saudi Arabia, [7][4][30] and most have limited findings as a result of the implementation of limited methodologies or the collection of data from limited samples. Therefore, the lack of sufficient and comprehensive studies that explore and analyse factors that influence the adoption and utilisation of e-Government in developing countries is considered an important challenge that needs to be addressed. The lack of utilisation of e-Services that are provided by eGovernment is problematic, even though some of these eServices have been implemented well. Implementing huge national systems, such as e-Government, involves a tremendous amount of effort and cost; however, unless it is utilised as expected, the result is a disaster. Thus, studies and research on investigating and analysing the significant factors that could influence the adoption and utilisation of e-Government in Saudi Arabia are becoming a necessity.

\section{THE RESEARCH MODEL}

In order to analyse factors that influence e-Government adoption and utilisation, this research developed a comprehensive model called the e-Government Adoption and Utilisation Model (EGAUM). This model provides a comprehensive framework to analyse key factors that have crucial influence on the utilisation and spread of e-Government. EGAUM was developed based on a critical analysis of the literature on technology acceptance, in conjunction with insights from several models and theories that are commonly used to analyse the acceptance and usage of technologies. Defects and shortcomings that exist in the models used in e-Government adoption literature are addressed in EGAUM. The main goal of this research model (EGAUM) is to determine factors that could influence the users' beliefs and intentions, as well as the behaviour that influences their adoption and usage levels.

The EGAUM model consists of three dependent variables, namely Intention to Use E-Government (ITU), E-Readiness of e-Government (ER) and Actual Adoption and Use of $e$ Government (AAU). EGAUM also contains four groups of independent variables, which are Personal Factors (PF), Motivational Factors (MF), Technical Factors (TF) and Reliability Factors $(R F)$. These independent variables represent the fundamental factors that have a critical influence on the adoption and usage levels of e-Government. The research model, EGAUM, is shown in Fig. 1.

The relationships between the research constructs are represented in two ways, namely by arrows (direct relationship) and small circles (indirect relationship). Direct relationship means that a construct has a direct influence. For example, Personal Factors $(P F)$ and Motivational Factors $(M F)$ have a direct influence on the Intention to use e-Government (ITU). Technical Factors $(T F)$ and Reliability Factors $(R F)$ also have a direct influence on the E-Readiness of e-Government (ER). However, an indirect relationship means that one or more factors of a specific construct has/have indirect influence; in other words, one or more factors of a construct can indirectly affect the influence of other constructs. For example, one or more of the Motivational Factors $(M F)$ indirectly affect the relationship between Personal Factors $(P F)$ and Intention to use e-Government (ITU). Moreover, one or more of the Personal Factors $(P F)$ has/have an indirect influence on the relationship between Technical Factors (TF) and the E-Readiness of $e$ Government (ER).

\section{A. Personal Factors (PF)}

It is proposed in this research that demographic factors such as the users' age, gender, education and computer literacy levels, as well as users' locations and income are factors 


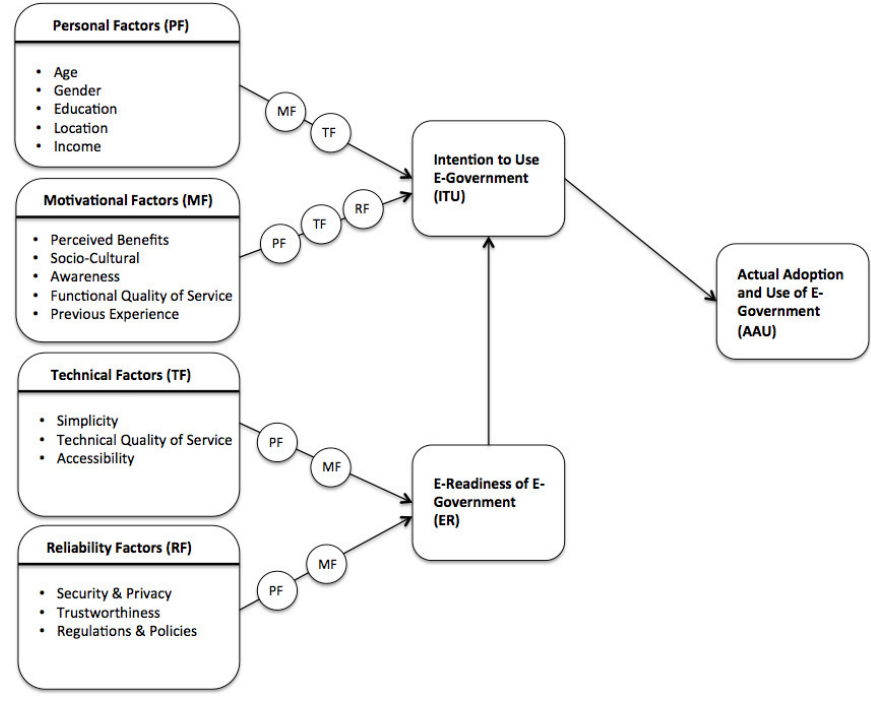

Fig. 1: The research model (EGAUM)

that potentially influence the adoption and utilisation of eGovernment. It is further suggested that all these previous factors have a direct influence on the intention to use eGovernment.

Age is an important factor that influences e-Government usage levels. Exploring the impact of the user's age on eGovernment adoption will lead to better understanding of agebased aspects that need to be improved in order to increase the adoption and utilisation of e-Government. Several studies have found that age is one of the important factors that influence the adoption of Information Technologies (IT), particularly in the Arab countries [31][32]. Moreover, various studies have revealed different results regarding the influence of age on the adoption of e-Government. An early study found that older users tend to correlate positively with the use of e-Government [33]. On the other hand, some recent studies have found that older users are less likely to use e-Services, and that users under 25 years of age are more likely to adopt and use eServices [34][35].

Gender is another crucial factor that needs to be addressed when analysing the adoption of e-Government systems, particularly in Saudi Arabia. Because of cultural and religious reasons, some Saudi governmental organisations and agencies allocate separate offices to serve women who need to perform governmental services physically. Although this division provides more privacy and comfort for the women and they are not as crowded as they would be in the main offices, these separate offices are not available in all cities, nor at all governmental organisations. This clearly influences the adoption and usage level on the part of women. Moreover, women are not allowed to drive cars in Saudi Arabia and there is insufficient public transport; thus, some women face difficulties when they need to go to government agencies. This also affects women's adoption and usage levels.

Education is also an important factor that needs to be in- vestigated when analysing e-Government adoption and usage. This is because of the strong correlation between computer and information literacy and the education level of a user [36]. Computer and information literacy is defined as everything a person needs to know in order to be able to use computers [37]. Users with higher levels of education are more likely to adopt and use e-Government systems because they know how to use the Internet and computers, even if they are not Information Technology specialists. On the other hand, users with low levels of education are unlikely to adopt and use e-Government.

Location is another important factor that is not covered in any of the previous research into the adoption of eGovernment. It is the right of all users to benefit from services that are provided by the government, regardless of whether they live in rural or urban areas; clearly, this poses a challenge for governments. In the context of Saudi Arabia, not all cities have governmental agencies and organisations that can provide governmental services for people who live in these cities. This challenge forces customers, who can be either citizens or business's representatives, to travel to the nearest city with the required governmental agency, a process that is both costly and time consuming. This will most likely have an effect on adoption and usage levels.

Income is another personal factor that will affect the intention to use e-Government systems. The EGAUM model included income as one of the Personal Factors (PF) that have a direct influence on the Intention to Use e-Government systems (ITU) because of two potential scenarios. The first suggested scenario is that income could prevent users from possessing computers and digital devices that could enable them to access e-Government services online, thus, preventing them from adopting and using e-Government systems. The second suggested scenario is that income could prevent users from travelling to the nearest city in the event that they live in rural areas without governmental offices in which to access governmental services physically. In both scenarios, the user's income is suggests a direct influence on the intention to use e-Government.

\section{B. Motivational Factors $(M F)$}

The Motivational Factors (MF) construct is one of the most important aspects to be investigated and analysed with regard to the adoption and utilisation of e-Government. This construct is comprised of four significant factors that are likely to influence the adoption and utilisation of e-Government.

Perceived Benefits in the E-Government Adoption and Utilisation Model (EGAUM) can be defined as 'the degree to which e-Government provides functional and non-functional benefits to its users'. This is related to the belief in gaining benefits and the expected outcomes of conducting e-Services using e-Government systems. The idea is to analyse the influence of perceiving the advantages of using e-Government systems on the adoption and utilisation thereof. The Perceived Benefit factor is classified into two categories: 
- Functional benefits, which refer to 'any tangible benefits that a user can obtain from using the e-Government system'. These functional benefits include completing the intended services online from anywhere and at any time, synchronising and updating records among different governmental organisations, saving the history of conducted services, tracking the status of the conducted services, preventing bias from affecting the process of the conducted service and reforming the bureaucracy that exists in governmental procedures.

- Non-functional benefits refer to 'any intangible benefits that the user can obtain from using the e-Government system'. These non-functional benefits include time saving, cost saving, the comfort and convenience of conducting the service, avoiding crowds and long queues, reducing effort and independence, particularly for women in Saudi Arabia, as certain limitations exist.

Much of the research in the literature emphasised the importance of analysing the influence of the perceived benefits of a new technology on the adoption and diffusion of that technology [15] [16] [38] [39].

Socio-Cultural factor is also likely to have a strong impact on the implementation of a new technology, especially when that new technology is related to improving the lifestyle of society. It is a combination of two influential aspects, namely the social aspect and the cultural aspect. There is a strong correlation between them, since one aspect has the power to change the other. Social influence can be defined as "the normative pressure of associated members family or friends influences the intention to use e-government" [24]. Numerous research in the literature found that social influence, which is represented by friends, family members and colleagues, has a strong impact on the users' adoption level of a new technology, such as e-Government [40][41].

Cultural Influence can be defined as "the values, beliefs, norms and behavioural patterns of a group of people in a society for national culture, staff of an organisation for organisational culture, specific professions for professional" [42]. Cultural Influence has been widely investigated in the literature on the adoption of technologies, and it is proposed that cultural norms have a fundamental correlation with the intention to use e-Government systems [43] [44]. Many aspects of social and cultural influences need to be investigated and analysed in order to discover their impact on the adoption and usage of e-Government systems. The most important aspects that are suggested in this research can be explained in the following points:

- Image : This refers to the users' perceptions that using and adopting an e-Government system will afford them societal superiority. It is claimed in the literature that adoption of e-Government might reflect the adopter's familiarity with modern technologies, his or her proficiency in using computers and the Internet, a high degree of modernism and a higher level of education. These phenomena add a degree of prestige and social value to the adopter [20]. This research suggests that this phenomenon is even more influential in Saudi society.

- Influence of others : It is now widely accepted that "the most powerful influence on human behaviour is other people" [45]. The power of the social influence runs very deeply, since whom we know and how we feel about others affects our behaviour. In the Saudi context, the impact of others is even more influential, since the society in the Kingdom of Saudi Arabia is interrelated and coherent. Most of the relationships between family members, as well as the social relationships between different families and friends, are strong.

- Resistance : The adoption and utilisation of any new technology is greatly affected by the behavioural norms of the society, since individuals may tend to resist changes that technologies can make, which is likely to lead to negative consequences with regard to the implementation of such technology [46]. Resistance to change is considered one of the factors that can negatively affect the successful implementation of any system, including e-Government systems. Some users might resist using e-Government due to trust issues, whereas others might resist adopting eGovernment because of the face-to-face culture that is still influential in terms of customer confidence [47].

- The influence of interpersonal social networks (Connections or Wasta) : Wasta represents one kind of corruption in many government organisations in developing countries [47]. In Arabic, wasta means personal connections with certain employees or top managers who can accelerate processes or break rules in order to complete paperwork and conclude transactions. Since e-Government will limit such corruption because services will be treated electronically and all actions will be controlled, some customers (including citizens and businesses) may resist the use of e-Government and continue to seek help from their personal connections instead. Moreover, some employees may also resist using the e-Government system in order to retain their ability to break the rules when processing their relatives' applications.

Awareness of the functions and services that any interactive system can provide for its users is a very important factor. Perceived awareness is a strong contributor to the adoption of e-Government [20]. Thus, all governments that intend to implement the e-Government system, especially in developing countries, need to be conscious of making users aware of and familiar with e-Government, particularly users in remote areas. Not doing so is likely to create a severe digital divide and fail to achieve the goals of e-Government. The research also assumed that awareness can be improved in a variety of ways, such as interactive advertising, social media and traditional advertising methods. Social media can play a fundamental role in enhancing the adoption and utilisation of e-Government by increasing awareness levels in this regard. Some recent research has begun to analyse the impact of social media on e-Government interaction [48] [49]. 
Functional Quality of Service refers to the quality level of functional aspects of a service that is provided by eGovernment. Functional quality of service is a significant factor that must be studied from different aspects in order to achieve accurate and practical results. Delivery speed of and delivery options for required documents among government agencies and users are considered to be important aspects that can influence adoption and usage levels. Customer care is another important aspect of functional service quality and involves providing on-going technical support to customers, helping customers to perform e-Services, seeking customer feedback and ensuring their satisfaction. Other aspects of the functional quality of the services provided services are also likely to affect e-Government usage, such as providing different payment options for governmental transactions that involve fees, providing up-to-date information regarding eServices and making e-Services available 24 hours a day, seven days a week.

Previous Experience is another important factor that has been addressed in this research model (EGAUM). It refers to experiences that users have encountered in the past with regard to e-Services. In this research, two aspects concerning the influence of previous experience have been proposed. The first is previous experience of using online applications and services such as e-Business services, e-Commerce services, online banking, online shopping and online payments. The second is related to previous experience of using e-Services provided by the e-Government itself. Positive and negative results of using online services, either e-Government services or any other nongovernmental services, are likely to have a strong impact on future interactions with the e-Government. Furthermore, this factor will also influence the adoption and utilisation of e-Government when first using it, as well as the continued use thereof. The Motivational Factors (MF), which include the Previous Experience factor, have indirect influence on all other factors. This will determine the influence of all the research factors on the first use and on the continued use of e-Government.

\section{Technical Factors (TF):}

In any interactive information system, there will be technical aspects that need to be addressed and taken into account in order to achieve the desired goals of implementing such systems. Unlike certain research in the literature that focused on the technical infrastructure aspects of the e-Government system itself, such as the network infrastructure and required applications [44] [50], this research will focus on the theoretical technical aspects that are related to the users' adoption and interaction. The technical factors that are addressed in this research model (EGAUM) can be listed as:

Simplicity refers to those factors that can simplify the eGovernment system and make it easy to use. It has been established that the easier an information system is to use, a greater number of users will adopt it. This relationship is particularly applicable and important with regard to large systems such as e-Government, as large numbers of users are expected to utilise it, and their skills and abilities may differ significantly. Simplicity includes the following factors:

- E-Government interface design:

E-Government's websites and interfaces are the interaction mediator between the e-Government system and its users. Therefore, they must be designed according to the users' requirements in order to achieve satisfactory levels of utilisation. E-Government interface design includes web page layout, design consistency, colour contrast, being free of spelling errors, having a clear font, clear labels, searchable contents and multiple language options.

- Reaching e-Services:

This refers to how easy it is to reach to the intended e- Service on the e-Government website i.e. the ease of locating and navigating the intended e-Service after accessing the intended e-Government website. Difficulty in locating e-Services on e-Government websites is likely to have a negative effect on the adoption and utilisation of such e-Services, even if access to the e-Government websites is easy. Therefore, it is suggested in this research that if it is easier to reach e-Services, more e-Services will be performed; thus, the high adoption and utilisation thereof will be achieved.

- Service description and hints:

This factor refers to the descriptions of services that are used to explain the e-Service to the users, as well as how to apply for it, the requirements thereof, the way in which the e-Service will be processed and how it will be delivered. Such information provides the users with a better understanding of the methods involved in successfully implementing e-Services. Furthermore, providing tips and examples for conducting e-Services and information regarding the required information type is also important in assisting users to utilise e-Government services.

Technical Quality of Service is another form of quality of service that needs to be addressed when analysing the adoption and usage of e-Government systems. It refers to the technical aspects that are visible to the users and which can affect their willingness and intention to adopt and use the system. These technical aspects reflect the quality of the system, and they include:

- Suitability for people with special needs:

This refers to the e-Government system's ability to support features and technologies that can assist people with special needs to use and interact with the e-Government. Special needs include blindness, low vision, deafness, being hard of hearing and physical disabilities [51] [52].

- Free of syntax, semantic and linkage errors:

This refers to ensuring that e-Government system is free from technical errors such as broken links, payment confirmation errors, and server and network errors. Such errors are likely to lead to user frustration and dissatisfaction, resulting in low levels of adoption and utilisation [26]. 
Accessibility Accessibility is another technical factor that refers to the users' ability to access the e-Government system, and includes the available methods for reaching and accessing services provided by the e-Government system. Several important aspects that are related to e-Government accessibility will be addressed in this research. These important aspects include:

- The role of intermediaries in e-Government accessibility: Intermediaries can be defined as any private or public organisation that facilitates communication and coordination between public services providers and users [53]. Many users will be excluded from benefiting from eGovernment services due to certain access barriers, such as limited access to the Internet and computer illiteracy [54]. In this regard, Al-Madinah city, which is a large city in Saudi Arabia, has introduced so-called 'intermediary organisations' or 'e-offices', which operate under the legislation and authority of the Saudi government. They are physical intermediaries (organisations) that help people who have difficulty using e-Government. These difficulties include limited Internet access, computer illiteracy and the inability to pay online. Al-Sobhi et al. have studied the influence of the intermediaries, and the results show that intermediaries are an extremely useful channel for improving the adoption and utilisation of eGovernment [24].

- Mobile e-Government:

With the spread and popularity of mobile communication devices, e-Government has become more accessible and adoptable in many countries [55]. Mobile communication devices include mobile phones, tablets and PDAs (Personal Digital Assistants). E-Government services are often difficult for users in remote areas to access, as well as being problematic for users that are homebound, or who have poor computer skills or chronic illnesses [56]. Thus, m-Government can enhance access for users with these conditions easily and efficiently, which will have a positive effect on the adoption and usage level.

\section{- Digital divide:}

The digital divide refers to the inequality between groups (individuals, households and businesses) in terms of access to and the use of Information and Communication Technologies (ICTs) [57]. Equality in terms of accessibility and the ability to use any information system is an important factor that can significantly affect the adoption and utilisation level. The importance of this factor is emphasised when the information system targets large populations and major users, such as e-Government systems.

\section{Reliability Factors $(R F)$}

The Reliability Factor (RF) construct is another important aspect of the EGAUM model that was developed in this research. This construct comprises fundamental factors that are related to the users' perceived risks and trust. These factors are:
Security and Privacy are two significant factors that need to be of a high standard in any interactive system. Their importance is emphasised when the system involves public users and sensitive data. In order to increase the adoption and usage level of information systems and applications, users need to feel safe when interacting with such systems. Implementing high standards of security and privacy is crucial, but it is not sufficient to increase the usage and utilisation level. Users need to be informed about the implementation of high security and privacy standards via the publication thereof, the sending of e-Services receipt confirmations, the requirement for further security criteria when performing financial transactions, and by informing the users when the e-Service requests the sharing of their personal information and requires permission to do so. Such factors are likely to influence perceptions of security and privacy, thereby increasing the users' adoption and utilisation of e-Government.

Trustworthiness plays a vital role in helping users to overcome the perceived risk and uncertainty involved in using online services. Trust issues can strongly affect the users' intention to share their personal information and to perform online transactions when using e-Government systems. Although trustworthiness has been studied and has proved to be an important factor in the literature on technology acceptance, there is still insufficient research that investigates and analyses the influence of trust on e-Government adoption and utilisation [30][58]. In this research, the trustworthiness factor will be investigated according to three aspects, namely trust in the Internet, trust in the e-Government system and trust in the eService provider. The importance of this factor is based on the fact that trust can be built from first impressions, and can also be affected by any later shortcomings. Moreover, trust is difficult to regain, particularly in an uncertain and virtual environment.

Regulations and Policies for using any interactive information system must be introduced and set up strictly and clearly in order to reach satisfactory levels of adoption and interaction. The importance of setting up clear, strict regulations is emphasised when the implementation of large public systems, such as the e-Government system, is involved. This factor includes several significant aspects, such as usage terms and conditions, e-Service delivery policies, payment policies, users' and providers' rights, data protection policies, and security and privacy policies. These examples of regulations must be introduced not only for the e-Service provider's records, but must also be published in order for the public users to be informed of their rights and to increase their confidence in the reliability of the system.

\section{Discussion}

As stated previously, this research model was proposed based on a critical analysis of the common technology acceptance models that have been utilised in most of the recent e-Government studies. A number of significant factors in these common models were integrated into this research model, albeit with broader insight. For example, the Perceived Benefits 
in EGAUM is similar to the concept of Perceived Usefulness in TAM and to Relative Advantages in DOI [15] [16], but has a wider interpretation. Moreover, the Simplicity factor is also more comprehensive than is the Ease of Use in TAM and the Effort Expectancy in UTAUT [15] [18].

On the other hand, several important factors in this research model were not addressed previously in most of the e-Government adoption and usage studies, especially those that were conducted in developing countries. This is because most of the e-Government adoption studies have utilised common technology acceptance models in the e-Government context that originally lacked many fundamental factors. These important factors have been added in this research model as independent factors, and include Cultural Influence, Personal Factors Influence, Awareness, Previous Experience Influence, Functional and Technical Quality of Service, Security and Privacy, Regulations and Policies, and Trustworthiness.

We believe that this research model has proposed and developed a simplified and comprehensive manner for providing a scientific framework for the analysis of e-Government interaction. Moreover, EGAUM is a universal model, which means that it can be utilised in countries other than Saudi Arabia. It can also be adapted and used to analyse the adoption and utilisation of different interactive systems and various service applications because it addresses influential factors that are crucial in such an analysis. EGAUM is currently being tested and validated to explore the influence of its constructs on e-Government adoption and utilisation in Saudi Arabia.

\section{CONCLUSION}

In order to investigate and analyse the key factors that can influence users' adoption and utilisation of e-Government services in Saudi Arabia, this research proposed a comprehensive and conceptual model (EGAUM) that would be the basis of our future work. A number of significant factors that have been integrated into this research model were taken from well-studied technology acceptance models in the existing literature. Other crucial factors have been developed and added in this research in order to conclude a model for e-Government adoption and utilisation analysis that is more comprehensive and appropriate.

The EGAUM model is now being validated and tested on various user groups, including public users of e-Government services, office workers that provide e-Government services and business managers who use e-Government services as part of their business activities, in order to determine the direct and indirect influences of the model's constructs on actual eGovernment adoption and utilisation.

\section{REFERENCES}

1] R. Davidrajuh, Planning e-government start-up: a case study on e-Sri Lanka. Electronic Government: An International Journal, Volume 1, No 1, pp. 92-106, 2004.

2] S. Alshafi and V. Weerakkody, Factors affecting e-government adoption in the state of Oatar. Proceedings of the European and Mediterranean Conference on Information Systems, Abu Dhabi, UAE, 2010.
[3] S. Archmann, and J. Castillo Iglesias, eGovernment: A Driving Force for Innovation and Efficiency in Public Administration. EIPAScope, 2010 (1). pp. 29-36.

[4] T. Gebba, and M.R. Zakaria, E-Government in Egypt: An Analysis of Practices and Challenges. International Journal of Technology and Management. Vol. 1 No. 1, pp. 11-25, 2012.

[5] Y. Kitaw, E-Government in Africa Prospects, Challenges and Practices. Swiss Federal Institute of Technology, 2006.

[6] U.N., Benchmarking E-government: A Global Perspective. United Nation Division for Public Economics and Public Administration American Society for Public Administration: USA, 2001.

[7] S. Alateyah, R. Crowder, and G. Wills, Citizen Adoption of Egovernment services. International Conference on Information Society. University of Southampton, United Kingdom, 2012.

[8] A. Grönlund, INTRODUCING e-GOV: HISTORY, DEFINITIONS, AND ISSUES. Communications of the Association for Information Systems, Volume 15, 2004

[9] E. Ziemba, T. Papaj, and R. Zelazny, A Model of Success Factors for E-Government Adoption - The Case of Poland. Issues in Information Systems, 14(2), pp. 87-100, 2013.

[10] Z. Ebrahim, and Z. Irani, E-government adoption: architecture and barriers. Business Process Management Journal, Vol. 11 Iss: 5, pp.589611, 2005. DOI: 10.1108/14637150510619902

[11] M. Alshehri, and S. Drew, Challenges of e-Government Services Adop tion in Saudi Arabia from an e-Ready Citizen Perspective. World Academy of Science, Engineering and Technology, 2005.

[12] Z. Al-adawi, S. Yousafzai, and J. Pallister, Conceptual model of citizen adoption of E-government. The Second International Conference on Innovations in Information Technology, 2005.

[13] S. Sahraoui, E-government in the Arabian Gulf: government transformation VS. government automation. eGovernment Workshop, Brunel University, West London, 2005.

[14] I. Ajzen, and M. Fishbein, Belief, attitude, intention, and behaviour: An introduction to theory and research. Reading, MA: Addison-Wesley, 1975

[15] D. Davis, P. Bagozzi, and R. Warshaw, User Acceptance of Computer Technology: A Comparison of Two Theoretical Models. Management Science, Vol. 35, No. 8, 1989.

[16] E. Rogers, Diffusion of Innovations. The Free Press, New York, USA, 1995

[17] G. Moore, and I. Benbasat, Development of an instrument to measure the perceptions of adopting an information technology innovation. Information Systems Research, Vol. 2 Issue 3, pp192, 1991. DOI: 10.1287/isre.2.3.192

[18] V. Venkatesh, M. Morris, G. Davis, and F. Davis, User Acceptance of Information Technology: Toward a Unified View. Management Information Systems Quarterly, Vol. 27, Issue.3, 2003.

[19] S. Sang, and J.D. Lee, A Conceptual Model of e-Government Acceptance in Public Sector. Third International Conference on Digital Society, Washington, DC, USA, pp. 71-76, 2009.

[20] M. Shareef, V. Kumar, U. Kumar, and Y.K. Dwivedi, $e$ Government Adoption Model (GAM): Differing service maturity levels. Government Information Quarterly. 28, 17 - 35, 2011. DOI: 10.1016/j.giq.2010.05.006

[21] E.M. Van Raaij, and J.J.L. Schepers, The acceptance and use of a virtual learning environment in China. Computers \& Education, vol. 50, no. 3 , pp. 838-852, 2008. DOI: 10.1016/j.compedu.2006.09.001

[22] M. Alshehri, S. Drew, and R. Alghamdi, Analysis of Citizens' Accep tance for e-Government Services: Applying The UTAUT Model. IADIS International Conferences, Theory and Practice in Modern Computing and Internet Applications and Research, pp. 69-76, 2012.

[23] A. Taiwo, A.K. Mahmood, and A.G. Downe, User Acceptance of eGOVERNMENT: Integrating Risk and Trust Dimensions with UTAUT Model. International Conference on Computer \& Information Science (ICCIS), 2012

[24] V. Weerakkody, R. El-Haddadeh, F. Al-Sobhi, M.M. Shareef, and Y.K. Dwivedi, Examining the influence of intermediaries in facilitating e-government adoption: An empirical investigation. International Journal of Information Management, 33, 716-725, 2013. DOI 10.1016/j.ijinfomgt.2013.05.001

[25] Yesser.gov.sa, E-Government program overview. [Online] Available from: http://www.yesser.gov.sa/en/ProgramDefinition/Pages/Overview. aspx (accessed in 20/01/14). 
[26] H. Al-Nuaim, An Evaluation Framework for Saudi E-Government Journal of e- Government Studies and Best Practices, IBIMA. Vol. 2011, 2011.

[27] KH. Al-Sabti, The Saudi Government In the Information Society, 2005. [Online] Available from: http://www.yesser.gov.sa/ar/mediacenter/ DocLib1/dubaiegov.pdf (accessed in 03/02/14).

[28] Yesser, The National e-Government Strategy and Action Plan, 2006. [Online] Available from: http://www.yesser.gov.sa/en/ MechanismsandRegulations/strategy/Pages/implementation_plan_ first.aspx (accessed in 25/10/13).

[29] M. Al-Suwail, E-government Program. Proceedings of the National eTransactions Conference, Al-Riyadh, Saudi Arabia, 2007.

[30] H. Alsaghier, M. Ford, A. Nguyen, and R. Hexel, Conceptualising Citizen's Trust in e-Government: Application of $Q$ Methodology. Electronic Journal of e-Government, Academic Conferences. Vol. 7, pp. 295-310, 2009.

[31] C.E. Hill, K.D. Loch, D.W. Straub, and K. El-Sheshai, A qualitative assessment of Arab culture and information technology transfer. Journal of Global Information Management, Vol. 6 No. 3, pp. 29-38, 1998.

[32] E. Baker, S. Al- Gahtani, and G. Hubona, The effect of gender and age on new technology implementation in a developing country. Information Technology and People, 20, 352-375, 2007. DOI: 10.1108/09593840710839798

[33] G, Sciadas, The Digital Divide in Canada. Ottawa: Statistics Canada, 2002.

[34] M. Al-Otaibi, and R. Al-Zahrani, Electronic commerce in the Kingdom of Saudi Arabia. King Saud University, Riyadh, 2009.

[35] K. Alrawi, and K. Sabry, E-commerce evolution: a Gulf region review. International Journal of Business Information Systems, 4, 509-526, 2009. DOI: $10.1504 /$ IJBIS.2009.025204

[36] F. Al-Sobhi, V. Weerakkody, and M.M. Kamal, An exploratory study on the role of intermediaries in delivering public services in Madinah City: Case of Saudi Arabia. Transforming Government: People, Process and Policy, vol. 4, pp. 14-36, 2010.

[37] M.K. Alomari, P. Woods, and K. Sandhu, Predictors for E-government Adoption in Jordan: Deployment of an Empirical Evaluation Based on a Citizen-centric Approach. Information Technology \& People, vol. 25, pp. 4-4, 2012. DOI: 10.1108/09593841211232712

[38] L.L. Tung, and O. Rieck, Adoption of electronic government services among business organisations in Singapore. Journal of Strategic Information Systems, 14, 417-440, 2005. DOI: 10.1016/j.jsis.2005.06.001

[39] L. Carter, and F. Belanger, The utilization of e-Government services: Citizen trust, innovation and acceptance factors. Information Systems Journal, 15(1), 5-25, 2005. DOI: 10.1111/j.1365-2575.2005.00183.x

[40] Z. Irani, Y.K. Dwivedi, and M.D. Williams, Understanding consumer adoption of broadband: An extension of the technology acceptance model. Journal of the Operational Research Society, 60, 1322-1334, 2009. DOI: $10.1057 /$ jors.2008.100

[41] V. Venkatesh, and S. Brown, A longitudinal investigation of personal computers in homes: Adoption determinants and emerging challenges. MIS Quarterly,25(1), 71-102, 2001. DOI: 10.2307/3250959
[42] K. Leung, R.S. Bhagat, N.R. Buchan, M. Erez, and C.B. Gibson, Culture and international business: recent advances and their implications for future research. Journal of International Business Studies, 36(4), pp. 357-378, 2005

[43] C. Akkaya, P. Wolf, and H. Krcmar, Factors Influencing Citizen Adoption of E-Government Services: A cross-cultural comparison. 45th Hawaii International Conference on System Sciences, 2012.

[44] S.A. Alateyah, R.M. Crowder, and G.B. Wills, Factors Affecting the Citizen's Intention to adopt e-Government in Saudi Arabia. World Academy of Science, Engineering and Technology Vol:81, 2013.

[45] D. Halper, Successful public service design must focus on human behaviour, The Guardian newspaper, 2013. [online] Available from: http://www.theguardian.com/public-leaders-network/2013/jan/17/ public-service-design-human-behaviour (accessed in 09/11/13).

[46] R.T. Watson, T.H. Ho, and K.S. Raman, Culture: A fourth dimension of group support systems. Communications of the ACM, 37(10): pp. 55 1994. DOI: $10.1145 / 194313.194320$

[47] S. AlAwadhi, and A. Morris, Factors Influencing the Adoption of Egovernment Services. Journal of Software, vol. 4, no. 6, 2009.

[48] H.M. Abdelsalam, C.G. Reddick, S. Gamal, and A. Al-shaar, Social media in Egyptian government websites: Presence, usage, and effectiveness. Government Information Quarterly, 2013.

[49] L. Zheng, Social media in Chinese government: Drivers, challenges and capabilities. Government Information Quarterly, 2013.

[50] F.H. Chanchary, and S. Islam, E-government Based on Cloud Computing with Rational Inference Agent. High Capacity Optical Networks and Enabling Technologies (HONET), pp.261-266, 2011.

[51] A. Abanumy, A. Al-Badi, and P. Mayhew, e-Government Website Accessibility: In-Depth Evaluation of Saudi Arabia and Oman. The Electronic Journal of e-Government Volume 3 Issue 3 pp 99-106, 2005.

[52] Alliance for Technology Access, Computer Resources for People with Disabilities: A Guide to Exploring Today's Assistive Technology. 2nd edition, Hunter House, 1996.

[53] M. Janssen, and B. Klievink, The role of intermediaries in the multichannel services delivery strategies. International Journal Of Electronic Government Research,5(3), 36-46, 2009. DOI: 10.4018/jegr.2009070103

[54] H. Margetts, and P. Dunleavy, Cultural barriers to e-government. Academic article for the report: 'Better Public Services Through egovernment'. London: National Audit Office, 2002.

[55] S.Y. Hung, C.M. Chang, and S.R. Kuo, User acceptance of mobile e-government services: An empirical study. Government Information Quarterly, 30, pp. 33-44, 2013. DOI: 10.1016/j.giq.2012.07.008

[56] S.A. Becker, Bridging Literacy, Language, and Cultural Divides to Promote Universal Usability of E-Government Websites. Northern Arizona University, 2009.

[57] M.D. Chinn, and R.W. Fairlie, The Determinants of the Global Digital Divide: A Cross-Country Analysis of Computer and Internet Penetration. Economic Growth Centre, Yale University, USA, Paper No. 881, 2004.

[58] P.A. Pavlou, and M. Fygenson, Understanding and predicting electronic commerce adoption: An extension of the theory of planned behaviour. MIS Quarterly,30(1), 115-143, 2006. 